\title{
COVID-19 Therapy With Mesenchymal Stromal Cells (MSC) and Convalescent Plasma Should Consider Exosome Involvement
}

\author{
Phillip W. Askenase, MD
}

\section{Corresponding Author:}

Phillip W. Askenase, MD, Professor of Medicine and Pathology, Section of Rheumatology and Clinical Immunology, Department of Internal Medicine, Yale University School of Medicine, 333 Cedar Street, New Haven Connecticut, 06520, USA, <philip.askenase@yale.edu>

\section{Short title:}

Exosomes from MSC and in Convalescent Plasma may give more optimal therapy to COVID-19 patients

\section{Keywords:}

Exosomes, Extracellular Vesicles, Mesenchymal Stromal Cells, MSC, miRNA, Cell Therapy, nanoparticles 


\section{Abbreviations:}

IV, intravenous administration,

MSC, mesenchymal stromal cells.

MSC exos, MSC-derived nano vesicle exosomes,

MHC, major compatibility complex,

GFP, green fluorescent protein,

Ab, Antibody

$\mathrm{Ag}$, antigen

\section{ABSTRACT}

Exosome extracellular vesicles as biologic therapy for COVID-19 therapy are discussed for two areas. The first involves growing use of mesenchymal stromal cells (MSC) for the profound clinical cytokine storm and severe pneumonia in Covid-19 patients. Instead, it is recommended to switch to treat instead with their MSC-released exosomes. This is because many reports in the literature of have shown definitively that the release of exosomes from the in vivo administered MSC is actually responsible for their beneficial effects. Further, the exosomes are superior, simpler and clinically more convenient compared to their parental MSC. Additionally, in the context of COVID-19, the known tendency of MSC to aggregate causing lung dysfunction might synergize with the pneumonia aspects, and the tendency of MSC peripheral vascular micro aggregates might synergize with the vascular clots of the COVID-19 disease process, causing significant central or peripheral vascular insufficiency.

The second involves use of COVID-19 convalescent plasma for its content of acquired immune antibodies that must consider the role in this therapy of billions of exosomes in the 
plasma. Many of these derive from activated immune modulating cells and likely transfer miRNAs that acting epigenetically to also influence the recipient response to the virus. These immune activated plasma exosomes may either be responsible for positive effects of the plasma beyond the contained immune antibodies, or could be inhibitory. Pre selection of plasma with the best antibodies and the best exosomes would produce the most optimum therapy for very severely affected COVID-19 patients.

\section{COVID 19 THERAPY WITH MESENCHYMAL STROMAL CELLS}

\section{Introduction to the wide clinical usefulness of mesenchymal stromal cells (MSC)}

MSC have been safely administered to humans in many clinical instances over the past 25 years. Commonly employed harvest sites for in vitro expansion of MSC precursors include bone marrow, adipose tissue, placenta, and umbilical cord. These antecedents of the employed MSC are present in these diverse connective tissue sites at a very low concentration; in the range of one per 10,000 cells $(0.001 \%) .1$

Since MSC are self-replenishing, adherent to culture surfaces and do not have specific surface markers of most immune and myeloid cells, they can be grown in vitro to huge numbers; up to $100 \%$ for in vivo use. Culture is performed on increasingly large surface areas of sterile flasks under strict aseptic conditions. The MSC and MSC exosome (MSC exos) phenotype is positive for MHC Class I, CD73, CD90, and CD105, and the exosomes also express typical surface marker tetraspanins (CD9, CD63 and CD81). They do not express typical T cell, B cell, macrophage, myeloid or embryonic cell surface marker antigens, and generally also no expression of MHC-II (HLA DR in humans) nor positive costimulatory surface molecules like include CD40, CD40L;1 overall suggesting immunomodulatory rather than effector properties.

Administering MSC intravenously (IV) to rodents with induced models of clinical diseases at just a million cells, can result in reversal of abnormalities for weeks thereafter. All told, these effects are nutritive, trophic, reparative, anti-inflammatory, and anti-immunologic; as well as healing micro vascular abnormalities. 2 Thus, regarding very ill patients with COVID-19 infections, MSC may be ideal for decreasing the pathogenesis of severe viral pneumonia and resulting acute respiratory distress syndrome (ARDS). In addition to infection, there are two accompanying potentially severe and life endangering immunologic syndromes that $\mathrm{MSC}^{\mathrm{exos}}$ may have special properties for beneficial 
treatment. The first is cytokine storm induced by the over reacting immune system, occurring in severe viral diseases.3,4 The second is emerging Kawasaki-like Multisystem Inflammatory Syndrome (MIS) of Children also resembling toxic shock syndrome.5,6,7

\section{Potential immune mediated Multisystem Inflammatory Syndrome as sequela of COVID-19 infection potentially benefitted by MSC therapy}

MIS in children is a seeming sequela of COVID-19 can occur after seeming recovery as a different delayed immune response. Clinically there is with unrelenting fever, skin rash with some of the following: non-purulent conjunctivitis or muco-cutaneous inflammatory edema (oral, hands or feet), generalized extremity pain and GI problems like diarrhea, vomiting, or abdominal pain. Systemically, there can be hypotension progressing to shock, refractory to volume resuscitation and eventually requiring inotropic support due to the dominant myocarditis with severe inflammation causing left ventricular dysfunction pericarditis, valvulitis, or coronary inflammation with aneurisms, eventuating into acute heart failure or cardiac arrest.6,7,8 Like toxic shock and cytokine storm there appears to be a diffuse in inflammatory process with development of small pleural, pericardial, and ascitic effusions.6 Laboratory tests show: elevated Troponin and cardiac enzymes, as well as evidence of coagulopathy (by PT, PTT, elevated d-Dimers) and markers of inflammation (ESR, C-reactive protein, or procalcitonin) without microbial infectious cause. Treatment with immune globulin, the previous approach to Kawasaki syndrome, can be associated with recovery of left ventricular systolic function. However, therapy with MSC or convalescent plasma has not previously been contemplated.

\section{Cytokine storm in severely ill patients infected with COVID-19 virus}

The cytokine storm occurs during primary active COVID-19 infection. It is due to rapid release of a variety of inflammatory cytokines from viral-activated immune system cells that are overreacting to the initial acute persistent viral infection. There are high fevers, edema, great muscle aches, extreme fatigue and nausea with multiple organ damage., Clinically there is rapid developing patchy bilobar pneumonia with pulmonary edema, dysfunctional air-exchange indicated by dangerously low pO2; all often progressing to (ARDS) induced by infection of alveolar cells. The pneumonia is visible as inflammatory lesions with ground-glass opacity on CT scan, with acute cardiac injury; in some leading to death.8a Considerable vascular abnormalities due to vascular injury and inflammation are induced by infection of the endothelium. 9 
This severe COVID-19 cytokine storm syndrome is primarily a virally triggered macrophage activation syndrome.10 Accordingly, there is preferential production of a variety of macrophage activating factors such as: IFN-g, its associated IP10 chemokine, monocyte chemoattractant protein 1 (MCP1) and monocyte chemoattractant protein 1 (MIP10); together with granulocyte-colony stimulating factor (GSCF), as well as IL-2, IL-6, IL-7, and TNFa.8a,9,11 The rises in macrophage directed cytokines are accompanied by severe lymphopenia; especially reduced $\mathrm{CD} 4^{+}$and $\mathrm{CD} 8^{+} \mathrm{T}$ cell counts. They induce pulmonary edema, dysfunctional of air-exchange with severe fall in pO2, acute respiratory distress syndrome, acute cardiac injury, and often secondary infection, leading to death. The severe $\mathrm{CD}^{+} \mathrm{T}$ cell depletion with bursts of IL- 6 and IL-8 cytokines are together particularly bad prognostic signs.12

\section{Numerous observations that MSC used for healing therapy are not found at their apparent site of action}

A crucial point is that when labeled MSC are given they seldom are found in the organ systems they are repairing. Numerous studies prove this is because they are primarily in the lungs, where they are eliminated by 24-48 hours after administration,12a and during which they release their MSC-derived exosomes (MSC ${ }^{\text {exos}}$ ) that actually are responsible for the healing processes they mediate.

MSC can be harvested from precursors in a variety of connective tissues greatly enriched by simple cultures for use as trophic and anti-inflammatory natural healing cells. It has been shown over the last several years that there is no evidence that MSC go to their targeted organs that were prospering.13

\section{Therefore, it is now incontrovertible, that healing, trophic and anti-inflammatory actions of administered MSC are due to their released exosomes (MSC ${ }^{\text {exos}}$ )}

Indeed, the therapeutic effects of systemic MSC can be replicated by systemic transfer of exosomes produced and secreted by the MSCs (MSC ${ }^{\text {exos}}$ ) in a variety of experimental injury models like myocardial infarction,14,15, with arrhythmia.16 Similarly in: CNS ischemic stroke,17,18 traumatic brain injury, 19,20 spinal cord injury,21 ischemia-reperfusion renal injury and dysfunction,22,23 liver drug injury, ischemia and fibrosis,24,25 lethal irradiation enteropathy,26 and hematopoiesis restoration, 27 and finally bronchopulmonary dysplasia28 and bleomycin pulmonary fibrosis.29,30 In these various experimental diseases, few, if any, of the systemically administered MSCs were 
detected at lesion sites that were healing and further, their derived exosomes were found to deliver almost identical healing.

Additionally, MSC ${ }^{\text {exos }}$ replacing their parental MSC modulate immune functions, 31,32 and consequently there is similar data as the above in models of autoimmune diseases; such as: Systemic Lupus,33,34,35 and the CIA model of rheumatoid arthritis.36,37 Type I Diabetes Mellitis38,39 Inflammatory bowel disease, 40 and the EAE model of multiple sclerosis.41,42,43

Finally, there are beginning studies of rodent models of MSC ${ }^{\text {exos }}$ therapy in Alzheimer's disease,44 Parkinson's' disease45 status elepticus46 and even autism.47 Therefore, it can be seen clearly that so called "cell therapy" with MSC is giving way to administration of their derived MSC ${ }^{\text {exos }}$. Further, it is also readily apparent that a very wide number of clinical tissue and organ injury diseases, as well as a variety of inflammatory, fibrotic and degenerative diseases have been found potentially susceptible to

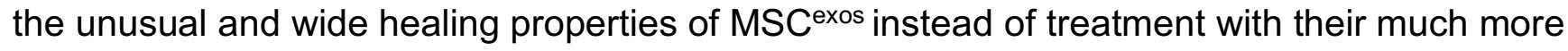
complicated parental MSC. It is our contention that this also applies to the use MSC ${ }^{\text {exos }}$ rather than the MSC in the treatment of patients with severe COVID-19 clinical syndromes.

\section{In MSC treatment of spinal cord injury (SCI), results show that MSC ${ }^{\text {exos }}$ mediate the tissue repair and functional recovery}

We confirmed the failure to find IV delivered MSCs at their apparent site of action in our studies of MSC-induced healing of spinal cord injury (SCI) in rats. I.V. injected fluorescent-labeled MSCs were not detected at lesion sites in the spinal cord, that none-the-less improved.21 I.V. infused green fluorescent protein (GFP)-expressing MSCs trafficked transiently to the lungs, spleen and liver, where they survived for only a few days.12a and none were detected within the contused spinal cord that was healing.21

This argued that the therapeutic effects are not mediated by MSC local replacement of damaged cells at the $\mathrm{SCl}$ site, nor by MSC paracrine effects at the site nor unlikely long distant $\mathrm{SCl}$-directed effects on local tissue repair at the site of injury. Instead, it indicates that the exosomes alone secreted by the MSC acted in an endocrine manner while stationed in SCl-distant organs like the lung and lymphoid organs. There, over a couple of days the MSC release MSC ${ }^{\text {exos }}$ into the general circulation, to then target and affect the distant damaged SCI tissues, to actually be responsible for the $\mathrm{SCI}$ healing and trophic effects. The proof was that the in vitro culture-derived MSC exos administered IV mediated all of 
the actions of the MSC in vivo and when they were labeled could be localized as preferentially targeting healing M2-type macrophages at the site of SCI.21

\section{The value of MSC and MSC-derived exosome clinical therapy in humans with a variety of diseases}

The above discussion dealt with properties of MSC and MSC exos in a large variety of disease models of experimental animals; a necessary prerequisite to human use. The natural initial dominance of clinical trials with MSC currently is reflected in the ten human disease trials listed at the NIH clinical trials.com site that include: diabetic nephropathy, multiple sclerosis, non-healing wounds and burns, lupus nephritis, allogenic transplantation, acute renal injury, refractory thrombocytopenia and liver failure. The more pertinent use of MSC exos instead is the we are considering here important we are considering here for therapy in appropriate severe COVID-19 patients considering here rather than MSC.

At the clinical trials.com site there also are ten projects listed using MSC ${ }^{\text {exos }}$ therapy; most in various cancers but one very interestingly testing MSC ${ }^{\text {exos }}$ aerosol treatment of normal volunteers. This certainly could be an antecedent to such therapy in patients with COVID-19 pneumonia that is not possible with cells like the MSC. It follows on phase I/II trials with human bone marrow-derived MSCs safely administered to patients with acute respiratory distress syndrome (ARDS) and septic shock,48 and pre clinical studies of Therapeutic Use of MSC exos in acute lung injury.49 Also, and very pertinent here, is study of therapy in ARDS in experimental influenza pneumonia, with aerosol application of exosomes directly into the COVID-19 affected lung.49a This is an application of the well known clinical fact that applying topical therapy at the disease site offers the best therapeutic index, as is done routinely with aerosol corticosteroids applied in allergy patients to atopic noses and asthmatic airways. Indeed, the airway exosomes that are released during influenza virus infection serve as a key component for stimulating the antiviral innate immune response.50 Thus, there is already is a considerable literature on the beneficial use of MSC in patients with a variety of diseases. Focusing on severe acute pulmonary disease as most pertinent here, patients with ARDS benefitted from their administration51,52,53 However, note that clinical trials of ARDS with MSC ${ }^{\text {exos }}$ has shown greater and more consistent benefit in part due to problems of variability of viability and effects among the MSC when compared, while the MSC exos have been much more consistent. Still there currently are many more worldwide clinical trials with the MSC, rather than with their seemingly superior derived MSC $^{\text {exos. }} 54$ 


\section{Use of MSC exos therapy in relevant human pulmonary diseases}

Thus, despite the numerous and growing data favoring replacement of MSC cell therapy with their produced MSC ${ }^{\text {exos }}$, that have been documented to actually be mediating their effects, some of the cognoscenti core of Mafia-like leaders of the MSC field, still in 2020, extol and focus on and invest in treatment employing the MSC. This results in numerous current companies still gearing up to deliver to patients raw MSC from various sources. This now seems to be a distinctly uninformed and inferior approach. It ignores the greater simplicity and most importantly the far less dangerous alternative use of the MSC-derived equally effective secreted MSC ${ }^{\text {exos }}$. However, the superior MSC exos alternative is now beginning to attract more research and commercial considerations with attempts to develop new methods for generation and isolation of MSC ${ }^{\text {exos }}$ that will simplify commercial use. Still, even in 2020, many studies persist that ignore the fact of exosome mediation of MSC effects, even when they consider the source of non-cellular trophic actions of MSC "secratome" factors.

\section{Use of MSC and MSC exos therapy in humans with COVID-19 pulmonary diseases.}

It is early days in the treatment of COVID-19 patients with MSC. In recent studies from China preliminarily tested MSC treatment of COVID-19 pneumonia.54a,54b,54c There was a remarkable reversal of disease and laboratory abnormalities within days of administering infusions of about $5 \times 10^{7} \mathrm{MSC}$ from allogeneic umbilical cord, with no toxicities. Also, there were accompanying decreases of inflammatory cytokine levels and increased anti-inflammatory factors and suppressive IL-10. MSC recovered from the patients showed high levels of anti-inflammatory and trophic factors including TGF $\beta$. These results and other facts about MSC, as reviewed here, have led to plans for MSC trials in COVID-19 patients.54d,54e, as already begun in several countries and as well for $\mathrm{MSC}^{\mathrm{exos}} . \mathbf{5 4 f}$

\section{There are a great number of advantages for the use of MSC ${ }^{\text {exos }}$ compared to therapy with their parental MSC.}

$\mathrm{MSC}^{\text {exos }}$ mitigate many safety concerns compared to use of viable replicating MSC. The cells may never really disappear in recipients, compared to the non-immortal exosomes. Further in the context of COVID-19, the know side effect that MSC IV administration may result in a coagulopathy55 with aggregating or clumping in the viral injured microcirculation,9 perhaps due to a complement related immune reaction to the abnormal endothelium.56 This can cause lung dysfunction that might synergize with the COVID-19 pneumonia effects. Similarly, the tendency toward similar MSCassociated peripheral vascular micro aggregates might synergize with the vascular clots of the COVID-19 disease process of causing central or peripheral vascular insufficiency leading to heart 
attack with cardiac arrest, stroke, or pulmonary embolism.57,58 In this time of hyper media coverage of COVID-19 virus, a few such cases would dissuade thousands from considering the MSC approach. As noted MSC exos have significantly less stimulatory HLA-complex molecules and surface costimulators.1 Thus, they are less innate immune stimulatory, nor acquired immunogenic, so that even allogeneic sources of MSC ${ }^{\text {exos }}$ have intrinsic tissue and cell preferential targeting/binding ability via their surface signature bar codes, compared to the MSC for which the peripheral tissues have no known receptivity.

$\mathrm{MSC}^{\text {exos }}$ are durable in culture for large scale manufacture, so that many can be harvested by repeated collections of conditioned media from the same adhering MSC donor cells in bioreactors with huge surface areas. Thus, MSC exos have reduced time and cost for producing therapeutic patient material. Further, biogenesis and secretion of the $\mathrm{MSC}^{\text {exos }}$ can be boosted several fold by incubation in neuro bioamines.59 They are non-viable but instead are subcellular particle organelles without functional genomic DNA, so they pose no risk of transferred abnormal chromosome numbers (aneuploidy), nor development of cancer promoting mutations and consequential oncogenicity.

The comparative safety of exosomes is emphasized by the fact that there are billions in blood plasma and patients have been receiving blood and plasma for many decades without problems,59a whereas MSC as cells are difficult to standardize or predict their actions in vivo. On a practical level MSCexos can be freeze-thawed at least once without toxic cryo-preservatives, with no lost activity for months to years, and it has been reported that they can be freeze-dried,60 for superior storage allowing on site administration without refrigeration. This can result in uncomplicated use on hospital wards or in poorer countries with low technology medical facilities. Thus, the MSC exos are available for immediate use post thawing without washing compared to cells for easier routine hospital use; they are an 'offthe-shelf' therapeutic with less manufacturing and storage costs.

MSC $^{\text {exos }}$ represent a thousand-fold purification as the actually effective component of MSC, via reduction in size and volume and have very different proteome and transcriptome compared to parental cells; obviously important considering that they are responsible for the biologic effects of the MSC that is most often med by the miRNAs that they deliver. As non-cells that are non-proliferating and not subject to differences in viability, $\mathrm{MSC}^{\text {exos }}$ can be more precisely standardized per dose and duration of biologic action, compared to the difficult variability of live MSC. Therefore, several reviews highly recommended that $\mathrm{MSC}^{\mathrm{exos}}$ hold great potential for targeted tissue repair.61,62,63,64,65 Over all, exosomes are simpler, safer, with lower cost, and more easy storage, transportation and use at 


\section{COVID-19 CONVALESCENT PLASMA LIKELY CONTAINS IMPORTANT EXOSOMES}

As noted, exosomes are nano vesicles made by all cells and are present in all body fluids. They contain greatly varying RNAs, proteins and lipid mediators that can alter the function of targeted cells near and far away via transit in the blood plasma. Especially important is that exosome miRNAs transferred to targeted acceptor cells can epigenetically alter functional properties since exosomes are the chief mechanism for regulation of the behaviors of different and distant cells genetically by such RNA transfers. Exosomes often act epigenetically by their transferred miRNA inactivating cytoplasmic mRNA encoding proteins that act to alter nuclear DNA by methylation and other means to change gene expression.

Exosomes in transit in the plasma have been studied in a variety of conditions because they can be markers of diseases, severity of disease and selection of therapies. Blood plasm exosomes are especially useful in cancer for "liquid biopsies" not only for diagnosis and prognosis, but also as mediating tumor induced resistance to routine radiation and drug therapies. As example here in COVID-19 ARDS patients, there can be a puzzle about the beneficial use and dose of corticosteroids and NSAIDs,66 in whom convalescent plasma exosomes might play a significant role. In fact, one study compared convalescent plasma to $1.5 \mathrm{gm}$ pulsed methylprednisolone in 19 vs. 21 SARS patients with progressive disease and found the plasma gave significantly better results.66a. Further the exosomes are undoubtedly an indicator of intercellular communications among the cells of the immune system to importantly serve towards choices for patient therapy.

\section{Exosomes may have been involved but unmeasured in favorable history of convalescent plasma therapy of viral diseases}

Prior positive findings concerning exosomes in convalescent plasma include serious RNA viral infections.66b Mostly beneficial effects were mostly poorly controlled recent studies of viral diseases antecedent to COVID-19; such as infections with related coronaviruses like SARS67,68 and MERS,69,70 as well as Ebola,71,72 avian influenza73 and influenza pneumonia74,75,76, with generally over all 60-80 \% improvement in severity or death rates. 
Three influenza studies deserve further mention. In one, 93 patients with severe pandemic influenza A (H1N1) 2009 virus infection, in a prospective cohort study were treated with convalescent plasma having a neutralizing antibody titer of $\geq 1: 160$, vs controls matched by age, sex, and disease severity.76a Mortality in the treatment group was significantly lower than in the nontreatment group ( $20.0 \%$ vs $54.8 \% ; P=.01)$ a $64 \%$ reduction. Subgroup demonstrated that plasma treatment was associated with significantly lower viral load, IL-6, IL-10 and TNFa vs. the control group $(P<.05)$.

Another study published recently (2019) examined anti-influenza immune plasma in patients with severe influenza $A$ in a prospective randomized, double-blind, phase 3 trial, comparing clinical efficacy of convalescent plasma with high titer anti-influenza $A$ antibodies to convalescent plasma with low titers.77 There was overall benefit but none in high titer vs. low titer plasma was found as in the prior phase 2 trial. This raises questions about the amount or class of Ab needed or other factors in the plasma such as perhaps strongly involved immune exosomes in both lots. Further, $34 \%$ of those treated experienced serious adverse events including ARDS and allergic transfusion reactions; highlighting the potential hazards of convalescent plasma.

The third study was a systematic review and meta-analysis of the effectiveness of convalescent plasma and hyperimmune immunoglobulin for the treatment of severe acute respiratory infections of viral etiology in several related studies published between 1918 and 1925 showing a 75\%\% reversal of case-fatality rate in the treated group vs the nontreated controls.78 Thus, this meta-analysis of the use of convalescent plasma in the pooled influenza and other virus studies sustained the positive conclusions of the individual past studies. Note also that in the previous early twentieth century convalescent sera and plasma were used to seemingly be able to stem outbreaks of viral diseases such as poliomyelitis, $\mathbf{7 9}$, measles, $\mathbf{8 0 , 8 1}$ mumps $\mathbf{8 2}$, and a meta-analysis of studies using convalescent blood products to treat the Spanish influenza pneumonia of 1918-19.83

\section{Unusual properties of exosomes possibly contributing to the positive effects of convalescent plasma}

Convalescent exosomes in general can Inhibit pathogen proliferation and transmission directly. They can allow the host to mount effective immune responses against pathogens, that includes activating antiviral mechanisms and transferring antiviral miRNAs derived from a variety of responding cells.66b In influenza virus infection, induced miRNAs in the exosomes regulate viral replication and have favorable influence on the host acquired immune response.84 
The structure of exosome membranes often changes as a consequence of infections, including alterations in the quantity of structural proteins and particularly and qualitive changes of constituent lipids influencing spatial configurations, resulting in particularly high viscosity that enables certain of

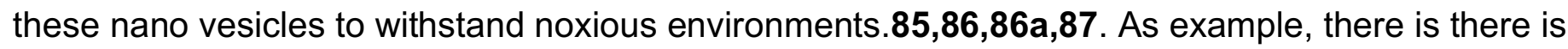
increasing evidence that immune activation of donor cells, as undoubtedly happens in viral illness due directly to the virus and the resulting immune response, alters membrane lipids to give some produced exosome subpopulations unusual features that may have great relevance to their role in the beneficial effects of convalescent plasma. As example, there is there is increasing evidence that immune activation of exosome donor cells, as undoubtedly happens in viral illness, due directly to the virus and to the resulting immune response. This alters membrane lipids of some produced exosome subpopulations unusual features that may have great relevance to their role in the beneficial effects of convalescent plasma.

This activation can include exosomes with unusual resistance that allows them to function in noxious environments like the acid/enzymes of the stomach and in hypoxic environments; both of which likely apply to affected pulmonary tissue in COVID pneumonia and ARDS. Such "activated" exosome subpopulations from immune and perhaps viral activated cells are able to acquire particular surface abilities. These are ability to be systemically active after oral administration.88,89 Additionally, there is newly acquired ability to bind Ag-specific $A b$ free light chains.88,89,90 This can render suppressive $T$ cell-derived exosomes Ag-specific,90 enabling their surface anti-peptide $\mathrm{Ab}$ to bind peptide-Ag/MHC on targeted APC,89,91 for transferring the specific miRNA-150.88,89,92 This miRNA leads to inhibited production of cytokines like IFN- $\gamma$ by finally targeted Th1 T cells.93 This T cell subset is most important in pathogenicity of COVID-19 infection-associated cytokine storm.1,10

While inhibiting infection and suppressing deleterious aspects of the immune response of the cytokine storm, exosomes in convalescent plasma, particularly those from DC and B cells can also serve to augment the antiviral immune response by acting as miniature nano APC,94 (Rodrigues M, Fan J 2018). These can induce healing M2-type macrophages, 95 and promote generation of the particular subset among Th2 T cells that produce immunosuppressive IL-10 and TGF- $\beta$. 1,10 A pertinent example is that exosomes used as vaccines containing the $S$ protein of the coronavirus pathogen of SARS pneumonia induce high levels of neutralizing antibodies.96 
Many things in biology and medicine acting in viral infections can function to favor the host or the pathogen. Exosomes can be tailored for function in either way by producing cells to alter target cell activities in many ways to then mediate their essential central function of delivering specific mediators. This applies especially to delivery of gene expression altering small RNAs that can function in both positive or negative directions to modulate the infection or the immune response depending on circumstances. They can promote immune resistance to viral infections in several ways; such as: inhibiting pathogen infection, proliferation and transmission directly, and indirectly Inhibiting Infection by Stimulating Immune resistance Responses by Improving functions of Macrophages, NK cells, T cells, and B cells.

Therefore, there are important functions that could be revealed in the study of patient plasma exosomes in COVID-19 infection that would bear consideration in selection of particular plasmas for therapy according to their potential positive effects on the infection and on the infection associated deleterious immune responses like ARDS and the coagulopathy, as well as the anti-viral Ab titers.

\section{Potential beneficial functions of the exosomes in convalescent plasma of patients with COVID-19 infections}

Up to now there have been few observations of exosomes in the plasma of patients with the COVID19 infections and its syndromes. In adults, plasma blood tests early in COVID-19 infections for studying exosome phenotype, proteome and transcriptome might be useful as "a liquid biopsy" in diagnosing those patients that will go on to severe sudden pneumonia and the need for artificial ventilation, as well as predicting the likelihood and extent of the cytokine storm syndrome. Similar analysis of plasma exosomes might be useful in distinguishing the pediatric patients that will go on to have the severe Kawasaki Disease-like MIS affecting the heart and great vessels.

Per treatment, firstly COVID-19 convalescent plasma analysis of contained anti-viral antibodies is thought useful for selecting particularly strongly therapeutic lots for treatment of the infections and/or induced associated syndromes. This would be based on in vitro assay of the acquired infectionassociated viral antigen-specific lgG antibody titers against the virus that if used for treatment can help block or clear the infectious agent early in the illness, and is hoped to be useful additionally for treating the viral and host response mediated pathology.

Secondly, the use of COVID-19 convalescent plasma for its content of acquired immune antibodies must also consider the role in this therapy of the billions per milliliter of exosomes contained in the 
plasma. These likely mediate functions of viral activated immune modulating cells due transferred miRNAs acting epigenetically. These immune activated plasma exosomes may be importantly involved in the positive effects of the plasma beyond the contained immune antibodies, or could possibly be inhibitory. Pre selection of plasma with the best antibodies and the best exosomes would produce the most optimum convalescent plasma therapy for very severely affected COVID-19 patients.

Before the development of antibiotics in the 1930s and1940s convalescent patient plasma or serum of intentionally immunized animals were the only organism-specific mode for treatment of infections like pneumonias.97,98 Moreover, now for the first time in the use of convalescent plasma, it is realized that in addition to antibodies against the infectious agent, it also contains biologically active exosomes in billions per milliliter. Experimental work in other pandemics suggests that these nano vesicles could also be powerful inhibitors of destructive pneumonias and the excessive immune response that is driving the over blown cytokine storm of severe in severe infections like with COVID-19.

It will be at the least several months or more until really effective drug treatment of active infection will be available to replace convalescent plasma. Additionally, even longer time must elapse until effective vaccines are available to prevent infection. Therefore, for now and many months ahead, given the large continuous number of new patients and the substantial severity and death rate, use of convalescent plasma for its antibody content, will be the only viral-specific treatment available. Appropriately, there are developing individual and inter connected consortium programs with active participation of professional statisticians and experts on designing controls, and for organizing and directing important large scale trials. These will be aimed hopefully at reaching routine use of convalescent plasma for treating hospitalized active cases of COVID-19 infections with severe complications. In the current rush to find effective treatments for such severe current COVID-19 patients, studies are on the way to use convalescent plasma for treatment in which the entire focus is on the contained immune antibodies; with no planned consideration of the important and perhaps crucial exosome aspect.

In contrast, relevant drugs being used today and contemplated are non-specific blockers of limited steps among many constituting the innate and acquired host immune response producing the cytokine storm. These are focused on blocking the IL-6 receptor and signaling, as well as other cytokines,99,100 or required viral signaling pathways infected host cell. 101,102,103 
As programs for administering COVID-19 convalescent plasma are being comprehensively and hurriedly generated to meet the masses of potential patient recipients, investigators say it is some time before they can even think about the role of the exosome content of the plasma. In 2020 this is a wrong decision about an important element of the plasma therapies never before contemplated since the presence and likely large effects of plasma exosomes was not known in all prior studies.

\section{Potential roles of exosomes in Covid-19 convalescent plasma derived from viral and immune induced host cells}

To my view considering the effects of the exosomes in the convalescent plasma needs to be done sooner rather than later. Looking over prior knowledge of exosomes and especially their role in immune regulation, it seems that plasma during COVID-19 infection, and especially use in those with the cytokine storm and ARDS may be augmentative as likely derived from the over blow innate immune cell response of the macrophage family per Mac 1-type subsets stimulated by TNF- $\alpha$ of the over blown innate immune response and subsequently during still active infection by the viral antigen activated Th1 cell and Th17 T cell-derived cytokines like INF- $\gamma$

With disease progression towards resolution and beyond, biology of the exosome response likely converts to reflect the healing positive aspect also in convalescent responses with more generation from the healing and trophic M2-type macrophages making IL-4, IL-10 and TGF- $\beta$, and perhaps later regulatory Th2 cells also making IL-10 and TGF- $\beta$, with IL-4, IL-13 and IL-25. Thus, convalescent plasma exosomes should be healing. Therefore, it is postulated that activated exosomes from immune stimulated regulatory and suppressor T cells and M2-type macrophages may make a very significant contribution to the beneficial effects of convalescent plasma. This would be beside and beyond the effects of remaining IgM antibodies of the primary immune response and the later developed crucial higher affinity IgG anti-COVID 19 antibodies of the acquired T cell mediated secondary B cell response. In fact, there may be effective Ag-specific Ab actually on the surface of the exosomes. $\mathbf{8 8 , 8 9 , 9 0 .}$

\section{A preclinical model with antigen-specific antibody coated suppressive exosomes}

\section{in the convalescent immune plasma}

In experiments in mice, repeated Ag high dose induced immune tolerance may be similar to experiencing the great and multiple viral Ag load in COVID-19 infection. In fact, tolerogenic suppression of the immune system is a regular part of many viral infections. 104,105,106 
In the preclinical high $\mathrm{Ag}$ exposure system, there is generated suppressive CD8 ${ }^{\text {pos }} \mathrm{T}$ cells that act by producing inhibitory anti-inflammatory and anti-immunological suppressive exosomes.107

These exosomes are Ag-specific due to a coating of specific $A b$ free light chains from neighboring activated $B$ cells, $89,89.90$ and are found in body fluids and importantly, also in the plasma.90 Like the separate protective plasma whole antibodies, these exosome-coating antibody light chains can be quite Ag-specific and by employing their specific binding to Ag-affinity chromatography are easy to isolate and strongly enrich.88,89,90.

This can provide a route to determining antibody variable-region gene sequences for development of biotechnology engineered monoclonal COVID-19 Ag-specific high affinity IgG antibodies for more definitive treatments that could be significantly developed before emergence of effective treating drugs or preventing vaccines. Further, determining the crucial suppressor exosome carried miRNAs responsible for the healing by the convalescent plasma exosomes via RNA sequence cloning may isolate crucial inhibitory miRNAs also useful in treating the patients. This convalescent-associated miRNA may lead to the cytosolic mRNA of targeted cells that they affect and subsequently on to the upstream DNA genetic sequence for possible development of molecular therapy for COVID-19 diseases as well. The exquisitely sophisticated currently available molecular methodologies may mean that these steps can be accomplished in a very short time, compared to the necessary much longer time to develop a safe, useful and effective vaccine or appropriate drug therapy.

Finally, in planning convalescent plasma therapy of COVID-19 patients, there will be a focus on determining the plasma samples with the most effective antibodies and titers using pre-testing with assays like in vitro viral neutralization. The argument here is that additional determination of the beneficial biologic effects of contained exosomes in the convalescent plasma should also be considered for constructing more optimal biologic therapy; adding to more precise selection of the most optimal plasma samples to use for the most effective treatment of very sick patients. Also, for some there will be a natural tendency to isolate the antibodies in the convalescent plasma to express potential therapeutic titers in $\mathrm{ug} / \mathrm{ml}$. There additionally will be refinements using sophisticated technologies aimed at isolating especially effective elite sub populations of the most effective broadly neutralizing antibodies as seemingly achieved for HIV,108 and the genes of the very few cells that produce them. However, this wholly antibody focus would mean blindly dispensing with the value of the similarly evolutionary optimized exosomes and thus potentially "throwing out the baby with the bath water." 


\section{Exosomes in convalescent plasma could be mediating negative therapeutic effects overall opposing the antibody beneficial effects}

Although there is much data suggesting that the exosome component of convalescent plasma likely contributes to the undoubted positive effects, like all things in biology there can be negative properties in some instances that may be detrimental when determined so that removing exosomes from the plasma may result in a better therapeutic agent for COVID-19 patients. Exosomes definitely can go in both directions dependent on the phase of illness. At the height of the cytokine storm and ARDS there is good reason to think that plasma exosomes mediate pro-disease effects. In convalescent plasma from later in the process, exosomes should have evolved properties to favor suppression of disease as reviewed here and thus be a useful aspect of convalescent plasma. However, the opposite may be true and thus to proceed we need to isolate and then then separate the exosomes from the $A b$ and remove them if this is so. Use of serum instead of plasma does not help since it contains most of the exosomes found in the plasma.

There is a paucity of knowledge about the clinical effects of exosomes in convalescent plasma, and no data are yet available about this aspect of COVID-19 infections. There definitely can be negative effects of exosomes induced during the infection as the virus uses host production of exosomes for its benefit. An important aspect is that virus stimulation of host cell exosome production favorable to thwarting the host is a central mechanism of how the virus controls the host. This might carry over into exosomes in the convalescent plasma and thus call for removal of the exosomes to improve the potential positive effects mediated by the acquired immune system production of beneficial convalescent antibodies. Further, like in suppression of the host effector T cell response in cancers we postulate that negative-acting exosomes in some of the potential COVID-19 therapeutic plasmas may contain suppressive exosomes surface PD-L1,109,110 or expressing other co-inhibitory receptors. These would be induced by the virus to thwart the host effector T cell mediated antiCOVID-19 response, and will have to be removed to obtain the best effect in treatment of the ARDS and cytokine storm processes. Finally, note that in EBOLA infections, induced exosomes can express a viral encoded antigen called VP40 that is a suppressor of effector T cells,111 and similarly would have to be removed. 


\section{Pilot largely uncontrolled treatment studies in 2020 of convalescent plasma for patients with severe COVID-19 syndromes}

In a half dozen preliminary reports of convalescent plasma treatment of severe COVID-19 published in early 2020 , the patients treated with $2-300 \mathrm{ml}$ convalescent plasma, have had no severe adverse effects with clearing of clinical parameters and improvement of cytokine levels in 1-3 days.. One study at heavily loaded Mt Sinai Hospital in New York City, with many available treated patients and disease controls involved 39 patients (the largest number treated), who were compared to carefully matched severe active disease controls, and strongly showed the value of convalescent plasma treatment. Namely, $12.8 \%$ of plasma recipients died compared to double that at $24.4 \%$ for the matched controls, with respectively $72 \%$ percent vs. $67 \%$ percent discharged alive.112 Together this and the other recent studies,113,114,115,116,117 show for now very strong evidence of the great value of convalescent COVID-19 plasma treatment, but leave open the important question concerning the role of antibodies compared to the contained exosomes.

An uncontrolled similar recent study of convalescent plasma therapy in 10 severe COVID-19 patients in China reported very similar clinical results with reduction of pulmonary chest CT lesions, increase in pO2 in all patients (indicative of recuperating lung function), and reversal of lymphocytopenia. In some patients there was resolution of COVID-2 viremia and increase in neutralizing antibody titers.116

\section{CONCLUSIONS}

These recent publications and many reported ongoing studies in several European countries, will result in more than a thousand patients treated with convalescent plasma by the summer of 2020,118 plus the later available results of large programs involving multiple thousands of patients and now recruiting under the FDA organized Convalescent Plasma Expanded Access Program administered through the Mayo Clinic, involving 40 leading US medical centers and in the UK as the NHS Plasma Program across its 23 main blood centers will provide definitive data to guide recommendations on the general role that convalescent plasma should have in the treatment of COVID-19 patients. For the huge Mayo Clinic Consortium study, as of June 1, 2020; 2400 sites and 7200 physicians were involved with 24,513 patients and 18,543 had been infused.

However, no current or planned studies include evaluating the role and planned participation on the contained exosomes. Certainly, compared to the much ballyhooed Remdesivir and now heavily invested in drug that acts on vial metabolism in which the major favorable result was just four days 
shorter hospitalizations, it is clear that convalescent plasma likely is far superior treatment. Surely in such an enormous effort there is room for and great need and it is strongly recommended that a major effort be mounted to determine the role of and relative strengths of contained antibodies vs. carried exosomes; the latter potentially delivering important anti-viral and pro-host epigenetic function altering miRNAs.

\section{SUMMATION}

There was a recent editorial by Dr. Sunny Dzik of the MGH, who is the editor of Transfusion Medical Review, entitled: "COVID-19 Convalescent Plasma: Now Is the Time for Better Science."119 I agree completely with his arguments that after almost ten instances of use of convalescent plasma in epidemics and pandemics over more than a century we are now about to use convalescent plasma requiring at long last thoroughly conceived and controlled studies to truly establish efficacy; minimally at least a the level of "first do no harm." I add bringing attention to the fact that convalescent plasma undoubtedly contains very biologically active exosomes mediating important aspects of the effects of this plasma therapy that were not known of previously, and so far are to be ignored in currently planned better scientifically conceived studies. All told, it is committing medial research malpractice to use MSC instead of the their produced MSC exos that are responsible for their actions. It also can be seen as medical research malpractice to perform studies of convalescent plasma treatment aimed entirely at the antibody content, avoiding the "Elephant in the Room." These companion immune and viral activated exosomes in billions per milliliter, that undoubtedly play an important role in the effects of the plasma, may be crucial to the outcome of convalescent plasma treatment of very sick patients with COVID-19 syndromes.

\section{ACKNOWLEDGEMENTS}

I am indebted to Drs. Krzysztof Bryniarski and Katarzyna Nazimek, and the late Professor Wlodzimierz Ptak for the great amount of related experimental work they have done over many decades presented here in part, and for discussions with them that stimulated some of the ideas expressed here. This work was supported by grants No Al-076366, Al-07174, and Al-1053786 from the National Institutes of 
Health. The author is indebted to Dr Irwin Braverman at Yale for much help with increasing the clarity of expression in the manuscript.

\section{CONFLICT OF INTEREST}

None

\section{REFERENCES}

1. Saeedi $P$, Halabian R, Imani Fooladi AA. A revealing review of mesenchymal stem cells therapy, clinical perspectives and Modification strategies. Stem Cell Investig. 2019;6:34. doi:10.21037/sci.2019.08.11

2. Andrew M. Hoffman, Steven W. Dow, Concise Review: Stem Cell Trials Using Companion Animal Disease Models. Stem Cells, Volume34, Issue7, July 2016, Pages 1709-1729

3. Huang KJ, Su IJ, et al. An interferon-gamma-related cytokine storm in SARS patients [J]. J. Med. Virol. 2005 Feb;75(2).

4. Mehta P, McAuley DF, Brown M, et al. COVID-19: consider cytokine storm syndromes and immunosuppression. Lancet. 2020;395(10229):1033-1034.

5. Lucio Verdoni, Angelo Mazza, Annalisa Gervasoni, Laura Martelli, Maurizio Ruggeri, Matteo Ciu reda, Ezio Bonanomi, Lorenzo D'Antiga. An outbreak of severe Kawasaki-like disease at the Italian epicentre of the SARS-CoV-2 epidemic: an observational cohort study. The Lancet. May 13,2020

6. Riphagen S, Gomez X, Gonzalez-Martinez C, Wilkinson N, Theocharis P. Hyperinflammatory shock in children during COVID-19 pandemic. Lancet. 2020;395(10237):1607-1608.

7. Schroeder AR, Wilson KM, Ralston SL. COVID-19 and Kawasaki disease: finding the signal in the noise. Hosp Pediatr. 2020; doi: 10.1542/hpeds.2020-000356

8. Zahra Belhadjer, Mathilde Méot, Fanny Bajolle etc. ....Pierre Mauran. Acute heart failure in multisystem inflammatory syndrome in children (MIS-C) in the context of global SARS-CoV-2 pandemic, $2020 \mathrm{https} / / /$ doi.org/10.1161/CIRCULATIONAHA.120.048360

8a. Mehta P., McAuley D.F., Brown M., Sanchez E., Tattersall R.S., Manson J.J. COVID-19: consider cytokine storm syndromes and immunosuppression. Lancet. 2020

9. Varga Z, Flammer AJ, Steiger P, et al. Endothelial cell infection and endotheliitis in COVID19. Lancet. 2020;395(10234):1417-1418 
10. McGonagle D, Sharif K, O'Regan A, Bridgewood C. The Role of Cytokines including Interleukin-6 in COVID-19 induced Pneumonia and Macrophage Activation Syndrome-Like Disease. Autoimmun Rev. 2020;19(6):102537.

11, Marveh Rahmati ${ }^{1}$, Mohammad Amin Moosavi ${ }^{2}$ Cytokine-targeted therapy in severely ill COVID-19 patients: Options and cautions. EJMO 2020;4(2):179-180

12.Zhang, X., Tan, Y., Ling, Y. et al. Viral and host factors related to the clinical outcome of COVID-19. Nature (2020).

12a. Matsushita T, Lankford KL, Arroyo EJ, Sasaki M, Neyazi M, Radtke C, Kocsis JD. 2015. Diffuse and persistent blood-spinal cord barrier disruption after contusive spinal cord injury rapidly recovers following intravenous infusion of bone marrow mesenchymal stem cells. Exp Neurol 267:152-64.

13. Yin, K., Wang, S. \& Zhao, R.C. Exosomes from mesenchymal stem/stromal cells: a new therapeutic paradigm. Biomark Res 7, 8 (2019).

14.Tan, S.J.O.; Floriano, J.F.; Nicastro, L.; Emanueli, C.; Catapano, F. Novel Applications of Mesenchymal Stem Cell-derived Exosomes for Myocardial Infarction Therapeutics. Biomolecules 2020, 10, 707

15. Sasse, S.; Skorska, A.; Lux, C.A.; Steinhoff, G.; David, R.; Gaebel, R. Angiogenic Potential of Bone Marrow Derived CD $133^{+}$and $\mathrm{CD} 271^{+}$Intramyocardial Stem Cell Trans- Plantation Post MI. Cells 2020, 9, 78.

16. Sadraddin, H.; Gaebel, R.; Skorska, A.; Lux, C.A.; Sasse, S.; Ahmad, B.; Vasudevan, P.; Steinhoff, G.; David, R. CD271+ Human Mesenchymal Stem Cells Show Antiarrhythmic Effects in a Novel Murine Infarction Model. Cells 2019, 8, 1474.

17. Xin H, Li Y, Chopp M. Exosomes/miRNAs as mediating cell-based therapy of stroke. Front Cell Neurosci. 2014;8:377.

18.Zhang, Z.G., Buller, B. \& Chopp, M. Exosomes - beyond stem cells for restorative therapy in stroke and neurological injury. Nat Rev Neurol 15, 193-203 (2019).

19. Xiong Y, Mahmood A, Chopp M. Emerging potential of exosomes for treatment of traumatic brain injury. Neural Regen Res. 2017;12(1):19-22.

20. Yang Y, Ye Y, Su X, He J, Bai W, He X. MSCs-Derived Exosomes and Neuroinflammation, Neurogenesis and Therapy of Traumatic Brain Injury. Front Cell Neurosci. 2017;11:55.

21. Karen L. Lankford, Edgardo J. Arroyo, Katarzyna Nazimek, Krzysztof Bryniarski, Philip W Askenase, and Jeffery D. Kocsis, Intravenously Delivered Mesenchymal Stem Cell-Derived Exosomes Specifically Target M2-type Macrophages of the Injured Spinal Cord. PLoS One. 2018 Jan 2;13(1):e0190358.

22. Li L, Wang R, Jia Y, Rong R, Xu M, Zhu, Exosomes Derived From Mesenchymal Stem Cells Ameliorate Renal Ischemic-Reperfusion Injury Through Inhibiting Inflammation and Cell Apoptosis. T.Front Med (Lausanne). 2019 Nov 19;6:269. 
23. Tsuji K, Kitamura S, Wada J. Immunomodulatory and Regenerative Effects of Mesenchymal Stem Cell-Derived Extracellular Vesicles in Renal Diseases. Int J Mol Sci. 2020;21(3):756. P2020.

24. Lou G, Chen Z, Zheng M, Liu Y, Mesenchymal stem cell-derived exosomes as a new therapeutic strategy for liver diseases. Exp Mol Med. 2017 Jun 16;49(6):e346.

25. Nong K, Wang W, Niu X, et al. Hepatoprotective effect of exosomes from human-induced pluripotent stem cell-derived mesenchymal stromal cells against hepatic ischemia-reperfusion injury in rats. Cytotherapy. 2016;18(12):1548-1559.

26. Chang PY, Qu YQ, Wang J, Dong LH. The potential of mesenchymal stem cells in the management of radiation enteropathy. Cell Death Dis. 2015;6(8):e1840.

27. Yang X, Balakrishnan I, Torok-Storb B, Pillai MM. 2012. Marrow Stromal Cell Infusion Rescues Hematopoiesis in Lethally Irradiated Mice despite Rapid Clearance after Infusion. Adv Hematol 2012:142530

28. Willis GR, Fernandez-Gonzalez A, Anastas J, et al. Mesenchymal Stromal Cell Exosomes Ameliorate Experimental Bronchopulmonary Dysplasia and Restore Lung Function through Macrophage Immunomodulation. Am J Respir Crit Care Med. 2018;197(1):104-116.

29. Nahal Mansouri, ... , S. Alex Mitsialis, Stella Kourembanas. Mesenchymal stromal cell exosomes prevent and revert experimental pulmonary fibrosis through modulation of monocyte phenotypes. JCI Insight. 2019;4(21):e128060.

30. Sharon Elliot, Gustavo Rubio, Xiaomei Xia, Paola Catanuto, Simone PereiraSimon, Joshua Shapiro, Marilyn K. Glassberg, Irena Pastar, Marjana TomicCanic, George Glinos, Therapeutic benefits of exosomes derived from mesenchymal stromal cells in bleomycin-induced pulmonary fibrosis in aged mice. European Respiratory Journal 2017 50: PA906;

31. Baquir B, Hancock RE. Exosomes, your body's answer to immune health. Ann Transl Med. 2017;5(4):81.

32. Chen W, Huang $Y$, Han J, et al. Immunomodulatory effects of mesenchymal stromal cellsderived exosome. Immunol Res 2016;64:831-40

33. Perez-Hernandez J, Redon J, Cortes R. Extracellular Vesicles as Therapeutic Agents in Systemic Lupus Erythematosus. Int J Mol Sci. 2017;18(4):717.

34. Sharma J, Hampton JM, Valiente GR, et al. Therapeutic Development of Mesenchymal Stem Cells or Their Extracellular Vesicles to Inhibit Autoimmune-Mediated Inflammatory Processes in Systemic Lupus Erythematosus. Front Immunol. 2017;8:526.

35. Zhou, T., Liao, C., Li, H. et al. Efficacy of mesenchymal stem cells in animal models of lupus nephritis: a meta-analysis. Stem Cell Res Ther 11, 48 (2020) 
36. Chen Z, Wang H, Xia Y, Yan F, Lu Y. Therapeutic Potential of Mesenchymal Cell-Derived miRNA-150-5p-Expressing Exosomes in Rheumatoid Arthritis Mediated by the Modulation of MMP14 and VEGF. J Immunol. 2018;201(8):2472-2482.

37. Li F, Li X, Liu G, Gao C, Li X. Bone Marrow Mesenchymal Stem Cells Decrease the Expression of RANKL in Collagen-Induced Arthritis Rats via Reducing the Levels of IL-22. J Immunol Res. 2019;2019:8459281.

38. Chang W, Wang J. Exosomes and Their Noncoding RNA Cargo Are Emerging as New Modulators for Diabetes Mellitus. Cells. 2019;8(8):853.

39. Nojehdehi $S$, Soudi $S^{3}$, Hesampour $A^{1}$, Rasouli $S^{4}$, Soleimani $M^{5}$, Hashemi $\mathrm{SM}^{6,7}$. Immunomodulatory effects of mesenchymal stem cell-derived exosomes on experimental type1 autoimmune diabetes. J Cell Biochem. 2018 Nov;119(11):9433-9443.

40. Fei Mao, Yunbing Wu, Xudong Tang, Jingjing Kang, Bin Zhang, Yongmin Yan, Hui Qian, Xu Zhang, and Wenrong Xu. Exosomes Derived from Human Umbilical Cord Mesenchymal Stem Cells Relieve Inflammatory Bowel Disease in Mice. Hindawi BioMed Research International. Volume 2017, Article ID 5356760, 12 pages

41. Riazifar M, Mohammadi MR, Pone EJ, et al. Stem Cell-Derived Exosomes as Nanotherapeutics for Autoimmune and Neurodegenerative Disorders. ACS Nano. 2019;13(6):6670-6688

42. Farinazzo, A., Angiari, S., Turano, E. et al. Nanovesicles from adipose-derived mesenchymal stem cells inhibit T lymphocyte trafficking and ameliorate chronic experimental autoimmune encephalomyelitis. Sci Rep 8, 7473 (2018).

43. Morteza Jafarinia, Fereshteh Alsahebfosoul, Hossein Salehi, Nahid Eskandari, Therapeutic effects of extracellular vesicles from human adipose-derived mesenchymal stem cells on chronic experimental autoimmune encephalomyelitis. J Cell Physiol, 2020; 1-12.

44. Reza-Zaldivar EE, Hernández-Sapiéns MA, Minjarez B, Gutiérrez-Mercado YK, MárquezAguirre AL, Canales-Aguirre AA. Potential Effects of MSC-Derived Exosomes in Neuroplasticity in Alzheimer's Disease. Front Cell Neurosci. 2018;12:317.

45. Jarmalavičiūtè $A^{1}$, Tunaitis $V^{2}$, Pivoraitè $U^{2}$, Venalis $A^{2}$, Pivoriūnas $A^{3}$. Exosomes from dental pulp stem cells rescue human dopaminergic neurons from 6-hydroxy-dopamine-induced apoptosis. Cytotherapy. $2015 \mathrm{Jul} ; 17(7): 932-9$.

46. Long Q, Upadhya D, Hattiangady B, Kim DK, An SY, Shuai B, Prockop DJ, Shetty AK. 2017. Intranasal MSC-derived A1-xosomes ease inflammation, and prevent abnormal neurogenesis and memory dysfunction after status epilepticus. Proc, Natl Acad Sci U S A 114:E3536E3545.

47. Alessio, N.; Brigida, A.L.; Peluso, G.; Antonucci, N.; Galderisi, U.; Siniscalco, D. Stem CellDerived Exosomes in Autism Spectrum Disorder. Int. J. Environ. Res. Public Health 2020, 17, 944. 
48. Abraham A, Krasnodembskaya A. Mesenchymal stem cell-derived extracellular vesicles for the treatment of acute respiratory distress syndrome. Stem Cells Transl Med. 2020; $9(1): 28-38$.

49. Lee JH, Park J, Lee JW. Therapeutic use of mesenchymal stem cell-derived extracellular vesicles in acute lung injury. Transfusion. 2019;59(S1):876-883.

40a. Khatri M, Richardson LA, Meulia T. Mesenchymal stem cell-derived extracellular vesicles attenuate influenza virus-induced acute lung injury in a pig model. Stem Cell Res Ther. 2018;9(1):17.

50. James G. Bedford ${ }^{1}$, Giuseppe Infusini ${ }^{2}$, Laura F. Dagley, Fernando Villalon-Letelier ${ }^{1}$, Ming Z. M. Zheng ${ }^{1}$, Vicki Bennett-Wood ${ }^{1}$, Patrick C. Reading ${ }^{1,3}$ and Linda M. Wakim, ${ }^{1 *}$ Airway Exosomes Released During Influenza Virus Infection Serve as a Key Component of the Antiviral Innate Immune Response, Front. Immunol., 12 May 2020

51. Zheng G, Huang L, Tong H, Shu Q, Hu Y, Ge M, Deng K, Zhang L, Zou B, Cheng B, Xu J. Treatment of acute respiratory distress syndrome with allogeneic adipose-derived mesenchymal stem cells: a randomized, placebo-controlled pilot study. Respir Res. 2014;15:39.

52. Wilson JG, Liu KD, Zhuo H, Caballero L, McMillan M, Fang X, Cosgrove K, Vojnik R, Calfee CS, Lee JW, Rogers AJ, Levitt J, Wiener-Kronish J, Bajwa EK, Leavitt A, McKenna D, Thompson BT, Matthay MA. Mesenchymal stem (stromal) cells for treatment of ARDS: a phase 1 clinical trial. Lancet Respir Med. 2015;3:24-32.

53. Shah T, Qin S, Vashi M, Predescu DN, Jeganathan N, Bardita C, Ganesh B, diBartolo S, Fogg LF, Balk RA, Predescu SA. Alk5/Runx1 signaling mediated by extracellular vesicles promotes vascular repair in acute respiratory distress syndrome. Clin Transl Med. 2018; 7:19. 2

54. Behnke J, Kremer S, Shahzad T, et al. MSC Based Therapies-New Perspectives for the Injured Lung. J Clin Med. 2020;9(3):682.

54a. Bing Liang, Junhui Chen, Tao Li, Haiying Wu, Wenjie Yang, Yanjiao Li, J., Li, Congtao Yu, Fangang Nie, Zhaoxia Ma, Mingxi Yang, Panrong Nie, Y. G., \& Chuanyun Qian, M. H. (2020). Clinical remission of a critically ill COVID-19 patient treated by human umbilical cord. chinaXiv, https://doi.org/10.12074/ 202002.00084.

54b. Shen C, Wang Z, Zhao F, et al. Treatment of 5 Critically III Patients With COVID-19 With Convalescent Plasma JAMA. 2020;323(16):1582-1589.

54c. Leng Z, Zhu R, Hou W, et al. Transplantation of ACE2- Mesenchymal Stem Cells Improves the Outcome of Patients with COVID-19 Pneumonia. Aging Dis. 2020;11(2):216-228.

54d. Atluri S, Manchikanti L, Hirsch JA. Expanded Umbilical Cord Mesenchymal Stem Cells (UCMSCs) as a Therapeutic Strategy in Managing Critically III COVID-19 Patients: The Case for Compassionate Use. Pain Physician. 2020;23(2):E71-E83.

54e. Golchin A, Seyedjafari E, Ardeshirylajimi A. Mesenchymal Stem Cell Therapy for COVID19: Present or Future. Stem Cell Rev Rep. 2020;16(3):427-433. 
54f. O'Driscoll L. Extracellular vesicles from mesenchymal stem cells as a Covid-19 treatment Drug Discov Today. 2020;S1359-6446(20)30170-7.

55. Fogarty H, Townsend L, Ni Cheallaigh C, et al. COVID19 coagulopathy in Caucasian patients Br J Haematol. 2020;10.1111/bjh.16749.

56. Magro C, Mulvey JJ, Berlin D, et al. Complement associated microvascular injury and thrombosis in the pathogenesis of severe COVID-19 infection: a report of five cases Transl Res. 2020;S1931-5244(20)30070-0.

57. Klok FA, Kruip MJHA, van der Meer NJM, et al. Incidence of thrombotic complications in critically ill ICU patients with COVID-19. Thromb Res. 2020;S0049-3848(20)30120-1.

58. Poissy, J. et al..... Sophie Susen. on behalf of the Lille ICU Haemostasis COVID-19 group. Pulmonary Embolism in COVID-19 Patients: Awareness of an Increased Prevalence. Circulation http://doi.org/10.1161/CIRCULATIONAHA.120.047430 (2020).

59. Wang J, Bonacquisti EE, Brown AD, Nguyen J. Boosting the Biogenesis and Secretion of Mesenchymal Stem Cell-Derived Exosomes. Cells. 2020;9(3):660.

59a. Michael Joyner, R. Scott Wright, DeLisa Fairweather, ... Arturo Casadevall. Early Safety Indicators of COVID-19 Convalescent Plasma in 5,000 Patients. doi: https://doi.org/10.1101/2020.05.12.20099879

60. El Baradie KBY, Nouh M, O'Brien lii F, et al. Freeze-Dried Extracellular Vesicles From Adipose-Derived Stem Cells Prevent Hypoxia-Induced Muscle Cell Injury. Front Cell Dev Biol. 2020;8:181.

61.Pashoutan Sarvar D, Shamsasenjan K, Akbarzadehlaleh P. Mesenchymal Stem Cell-Derived Exosomes: New Opportunity in Cell-Free Therapy. Adv Pharm Bull. 2016;6(3):293-299.

62. Phinney DG, Pittenger MF. MSC-Derived Exosomes for Cell-Free Therapy Stem Cells. 2017 Sep;35(9):2103]. Stem Cells. 2017;35(4):851-858.

63. Gowen A, Shahjin F, Chand S, Odegaard KE, Yelamanchili SV. Mesenchymal Stem CellDerived Extracellular Vesicles: Challenges in Clinical Applications. Front Cell Dev Biol. 2020;8:149.

64. Yin, K., Wang, S. \& Zhao, R.C. Exosomes from mesenchymal stem/stromal cells: a new therapeutic paradigm. Biomark Res 7, 8 (2019

65. Zhao T, Sun F, Liu J, et al. Emerging Role of Mesenchymal Stem Cell-derived Exosomes in Regenerative Medicine. Curr Stem Cell Res Ther. 2019;14(6):482-494.

66. Russell B, Moss C, Rigg A, Van Hemelrijck M. COVID-19 and treatment with NSAIDs and corticosteroids: should we be limiting their use in the clinical setting?. Ecancermedicalscience. 2020;14:1023.

66a. Soo YO, Cheng Y, Wong R, et al. Retrospective comparison of convalescent plasma with continuing high-dose methylprednisolone treatment in SARS patients. Clin Microbiol Infect. 
2004;10(7):676-678.

66b. Chahar HS, Bao X, Casola A. Exosomes and Their Role in the Life Cycle and Pathogenesis of RNA Viruses. Viruses. 2015;7(6):3204-3225.

67. Cheng $\mathrm{Y}$, et al. Use of convalescent plasma therapy in SARS patients in Hong Kong. Eur J Clin Microbiol Infect Dis. 2005;24(1):44-46.

68. Yeh KM, et al. Experience of using convalescent plasma for severe acute respiratory syndrome among healthcare workers in a Taiwan hospital. J Antimicrob Chemother. 2005;56(5):919-922

69. Ko JH, Seok H, Cho SY, et al. Challenges of convalescent plasma infusion therapy in Middle East respiratory coronavirus infection: a single centre experience. Antivir Ther. 2018;23(7):617-622.

70. Arabi YM, et al. Feasibility of using convalescent plasma immunotherapy for MERS-CoV infection, Saudi Arabia. Emerging Infect Dis. 2016;22(9):1554-1561.

71. Mulangu S, et al. A randomized, controlled trial of Ebola virus disease therapeutics. $\mathrm{N}$ Engl J Med. 2019;381(24):2293-2303.

72. Kraft CS, et al. The Use of TKM-100802 and convalescent plasma in 2 patients with Ebola virus disease in the United States. Clin Infect Dis. 2015;61(4):496-502.

73. Kong LK, Zhou BP. Successful treatment of avian influenza with convalescent plasma. Hong Kong Med J. 2006;12(6):489.

74. Luke TC, Casadevall A, Watowich SJ, Hoffman SL, Beigel JH, Burgess TH. Hark back: passive immunotherapy for influenza and other serious infections. Crit Care Med. 2010;38(4 suppl):e66-e73.

75. McGuire LW, Redden WR. The use of convalescent human serum in influenza pneumonia-a preliminary report. Am J Public Health (N Y). 1918;8(10):741-744.

76. Zhou B, Zhong N, Guan Y. Treatment with convalescent plasma for influenza A (H5N1) infection. N Engl J Med. 2007;357(14):1450-1451.

76a. Hung I.F., To K.K., Lee C.K., Lee K.L., Chan K., Yan W.W. Convalescent plasma treatment reduced mortality in patients with severe pandemic influenza A (H1N1) 2009 virus infection. Clin Infect Dis. 2011;52:447-456.

77.J.H. Beigel, E. Aga, M.C. Elie-Turenne, J. Cho, P. Tebas, C.L. Clark, et al. Anti-influenza immune plasma for the treatment of patients with severe influenza A: a randomized, doubleblind, phase 3 trial, Lancet Respir Med, 7 (2019), pp. 941-950 
78. Mair-Jenkins $\mathrm{J}$, et al. The effectiveness of convalescent plasma and hyperimmune immunoglobulin for the treatment of severe acute respiratory infections of viral etiology: a systematic review and exploratory meta-analysis. J Infect Dis. 2015;211(1):80-90.

79. Park WH. Therapeutic use of anti-poliomyelitits serum in preparalytic cases of poliomyelitis. JAMA. 1932;99:1050-1053.

80. Park WH, Freeman RG. The prophylactic use of measles convalescent serum. JAMA. 1926;87(8):556-558.

81. Gallagher JR. Use of convalescent measles serum to control measles in a preparatory school. Am J Public Health Nations Health. 1935;25(5):595-598.

82. Rambar AC. Mumps; use of convalescent serum in the treatment and prophylaxis of orchitis. Am J Dis Child. 1946;71:1-13. 83.

83. Luke TC, Kilbane EM, Jackson JL, Hoffman SL. Meta-analysis: convalescent blood products for Spanish influenza pneumonia: a future H5N1 treatment? Ann Intern Med. 2006;145(8):599-609.

84. Zheng B, Zhou J, Wang $\mathrm{H}$. Host microRNAs and exosomes that modulate influenza virus infection. Virus Res. 2020;279:197885.

85. Laulagnier K, Motta C, Hamdi S, et al. Mast cell- and dendritic cell-derived exosomes display a specific lipid composition and an unusual membrane organization. Biochem J. 2004;380 (Pt 1):161-171.

86. Li N, Huang Z, Zhang X, Song X, Xiao Y. Reflecting Size Differences of Exosomes by Using the Combination of Membrane-Targeting Viscosity Probe and Fluorescence Lifetime Imaging Microscopy. Anal Chem. 2019;91(23):15308-15316.

86a. Ning Li, Zhenlong Huang, Xinfu Zhang, Xinbo Song and Yi Xiao, Reflecting Size Differences of Exosomes by Using the Combination of Membrane-Targeting Viscosity Probe and Fluorescence Lifetime Imaging Microscopy Anal. Chem. 2019, 91, 23, 15308-15316

87.Zhou Q, Chen J, Luan Y, et al. Unidirectional rotating molecular motors dynamically interact with adsorbed proteins to direct the fate of mesenchymal stem cells. Sci Adv. 2020;6(5):eaay2756.

88. Magdalena Wąsik, Katarzyna Nazimek, Bernadeta Nowak, Philip W. Askenase, and Krzysztof Bryniarski. Delayed-Type Hypersensitivity Underlying Casein Allergy Is Suppressed by Extracellular Vesicles Carrying miRNA-150. Nutrients. 2019 Apr 23;11(4). pii: E907.

89. Krzysztof Bryniarski, Katarzyna Nazimek, Wlodzimierz Ptak, Tom Groot Kormelink, and Philip W. Askenase, Orally Administered T and B cell Antigen-Specific Suppressor Exosomes Deliver miRNA150 to Inhibit DTH Via Their Surface Antibody Light Chains Binding Antigen Peptides in MHC on APC Targeted Cells, Submitted 2020

90. Bryniarski K, Ptak W, Jayakumar A, et al. ....Askenase PW. Antigen-specific, antibody-coated, exosome-like nanovesicles deliver suppressor T-cell microRNA-150 to effector T cells to inhibit contact sensitivity. J Allergy Clin Immunol. 2013;132(1):170-181. 
91. Nazimek K, Ptak W, Nowak B, Ptak M, Askenase PW, Bryniarski K. Macrophages play an essential role in antigen-specific immune suppression mediated by $\mathrm{T} \mathrm{CD}^{+}$cell-derived exosomes. Immunology. 2015;146(1):23-32.

92. Krzysztof Bryniarski, Wlodzimierz Ptak, Emilia Sikora, Katarzyna Nazimek, Marian Szczepanik, Marek Sanak and Philip W. Askenase Free extracellular miRNA functionally targets cells by transfecting exosomes from their companion cells, PLoS One. 2015; 10(4): e0122991.

93. Tsuji RF, Szzcepanik, Kawikova I, Paliwal V, Campos RA, Itakura A, Akahira-Azuma M, Baumgarth $\mathrm{N}$, Herzenberg LA, and Askenase PW. B cell-dependent T cell responses. IgM antibodies are required to elicit contact sensitivity. J Exp Med 196:1277-1290, 2002

94. Meryl Rodrigues, Jia Fan, Christopher Lyon, Meihua Wan, Ye Hu. Role of Extracellular Vesicles in Viral and Bacterial Infections: Pathogenesis, Diagnostics, and Therapeutics. Theranostics 2018; 8(10):2709-2721.

95. Saha B, Kodys K, Adejumo A, Szabo G. Circulating and exosome-packaged hepatitis C singlestranded RNA induce monocyte differentiation via TLR7/8 to polarized macrophages and fibrocytes. J Immunol (2017) 198(5):1974-84.

96. Kuate S1, Cinatl J, Doerr HW, Uberla K, Exosomal vaccines containing the S protein of the SARS coronavirus induce high levels of neutralizing antibodies. Virology, 25 Jan 2007, 362(1):26-37

97. Horsfall FL, Goodner K, Macleod CM, Harris AH. Antipneumococcus rabbit serum as a therapeutic agent in lobar pneumonia. JAMA. 1937;108(18):1483-1490.

98. W. Barry Wood Jr. The role of the dosage of antiserum in the treat- ment of pneumococcal pneumonia. ii. the clinical application of the Francis skin test. J Clin Invest. 1940;19(1):105-121.

99. Marveh Rahmati ${ }^{1}$, Mohammad Amin Moosavi ${ }^{2}$. Cytokine-targeted therapy in severely ill COVID-19 patients: Options and cautions. EJMO. 2020; 4(2): 179-181

100. Felsenstein S, Herbert JA, McNamara PS, Hedrich CM. COVID-19: Immunology and treatment options. Clin Immunol. 2020;215:108448.

101. Catanzaro, M., Fagiani, F., Racchi, M. et al. Immune response in COVID-19: addressing a pharmacological challenge by targeting pathways triggered by SARS-CoV-2. Sig Transduct Target Ther 5, 84 (2020).

102. Gordon, D.E., Jang, G.M., Bouhaddou. et al. A SARS-CoV-2 protein interaction map reveals targets for drug repurposing. Nature (2020).

103. Yogendra Singh, Gaurav Gupta, Saurabh Satija, Kavita Pabreja. COVID -19 transmission through host cell directed network of GPCR (G protein-coupled receptors. Drug Dev Res 2020;1-3

104. Rong Ou, Menghua Zhang, Lei Huang, and Demetrius Moskophidis , Control of Virus-Specific CD8 T-Cell Exhaustion and Immune-Mediated Pathology by E3 Ubiquitin Ligase Cbl-b during Chronic Viral Infection. J Virology, Apr. 2008, p. 3353-3368 
105. Saeidi A, Zandi K, Cheok YY, et al. T-Cell Exhaustion in Chronic Infections: Reversing the State of Exhaustion and Reinvigorating Optimal Protective Immune Responses. Front Immunol. 2018;9:2569.

106. Bouhdoud L, Villain P, Merzouki A, Arella M, Couture C. T-cell receptor-mediated anergy of a human immunodeficiency virus (HIV) gp120-specific CD4(+) cytotoxic T-cell clone, induced by a natural HIV type 1 variant peptide. J Virol. 2000;74(5):2121-2130.

107. Nazimek K, Bryniarski K, Askenase PW. Functions of Exosomes and Microbial Extracellular Vesicles in Allergy and Contact and Delayed-Type Hypersensitivity. Int Arch Allergy Immunol. 2016;171(1):1-26.

108. Mendoza P, Gruell H, Nogueira L, et al. Combination therapy with anti-HIV-1 antibodies maintains viral suppression. Nature. 2018;561(7724):479-484.

109. Chen G, Huang AC, Zhang W, et al. Exosomal PD-L1 contributes to immunosuppression and is associated with anti-PD-1 response. Nature. 2018;560(7718):382-386.

110. Poggio M, Hu T, Pai CC, et al. Suppression of Exosomal PD-L1 Induces Systemic Anti-tumor Immunity and Memory. Cell. 2019;177(2):414-427.e13

111. Pleet ML, DeMarino C, Lepene B, Aman MJ, Kashanchi F. The Role of Exosomal VP40 in Ebola Virus Disease. DNA Cell Biol. 2017;36(4):243-248.

112. Sean T. H. Liu, Hung-Mo Lin, lan Baine, Ania Wajnberg, ...... Judith A. Aberg, Convalescent plasma treatment of severe COVID-19: A matched control study. medRxiv. doi: https://doi.org/10.1101/2020.05.20.20102236.

113. Chenguang Shen, Zhaoqin Wang, $\mathrm{PhD}^{1}$; Fang Zhao, $\mathrm{PhD}^{1}$; et al...... Lei Liu, $\mathrm{MD}^{1}$ Treatment of 5 Critically III Patients With COVID-19 With Convalescent Plasma. JAMA. 2020;323(16):1582-1589.

114. Ye M, Fu D, Ren Y, et al. Treatment with convalescent plasma for COVID-19 patients in Wuhan, China J Med Virol. 2020;10.1002/jmv.25882.

115. Zeng QL, Yu ZJ, Gou JJ, et al. Effect of Convalescent Plasma Therapy on Viral Shedding and Survival in COVID-19 Patients J Infect Dis. 2020;jiaa228. doi:10.1093/infdis/jiaa228

116. Kai Duan, Bende Liu, Cesheng Li, ..... Xiaoming Yang. Effectiveness of convalescent plasma therapy in severe COVID-19 patients. PNAS April 28, 2020117 (17) 9490-9496;

117. Qing-Lei Zeng, Zu-Jiang Yu, Jian-Jun Gou, Guang-Ming Li, ....Zhang-Suo Liu, Effect of Convalescent Plasma Therapy on Viral Shedding and Survival in Patients With Coronavirus Disease 2019, The Journal of Infectious Diseases, , jiaa228, https://doi.org/10.1093/infdis/jiaa228xxx

118. Cormac Sheridan, Convalescent serum lines up as first-choice treatment for coronavirus, Nature News, 07 May 2020

119. Dzik S. COVID-19 Convalescent Plasma: Now Is the Time for Better Science. Transfus Med Rev. 2020;S0887-7963(20)30026-2. 
